\title{
Anesthetic Management of a Patient With Charcot-Marie-Tooth Disease
}

\author{
Naohiro Ohshita, DDS, PhD,* Saeko Oka, DDS, PhD, $†$ Kaname Tsuji, DDS, PhD, $\neq$ \\ Hiroaki Yoshida, DDS, PhD, $\neq$ Shosuke Morita, DDS, PhD, $\ddagger$ Yoshihiro Momota, DDS, \\ PhD, $*$ and Yasuo M. Tsutsumi, MD, PhD $₫$ \\ *Department of Anesthesiology and $\ddagger$ First Department of Oral and Maxillofacial Surgery, Osaka Dental University, Osaka, Japan, $†$ Marunouchi \\ Dental Clinic, Kagawa, Japan, and §Department of Anesthesiology, Tokushima University, Tokushima, Japan
}

Charcot-Marie-Tooth disease (CMTD) is a hereditary peripheral neuropathy and is characterized by progressive muscle atrophy and motor-sensory disorders in all 4 limbs. Most reports have indicated that major challenges with general anesthetic administration in CMTD patients are the appropriate use of nondepolarizing muscle relaxants and preparation for malignant hyperthermia in neuromuscular disease. Moderate sedation may be associated with the same complications as those of general anesthesia, as well as dysfunction of the autonomic nervous system, reduced perioperative respiratory function, difficulty in positioning, and sensitivity to intravenous anesthetic agents. We decided to use intravenous sedation in a CMTD patient and administered midazolam initially and propofol continuously, with total doses of $1.5 \mathrm{mg}$ and $300 \mathrm{mg}$, respectively. Anesthesia was completed in 3 hours and 30 minutes without adverse events. We suggest that dental anesthetic treatment with propofol and midazolam may be effective for patients with CMTD.

Key Words: Charcot-Marie-Tooth disease, Intravenous sedation, Propofol

$\mathrm{C}$ harcot-Marie-Tooth disease (CMTD) was described independently by J. M. Charcot, P. Marie, and H. $\mathrm{H}$. Tooth in $1886 .{ }^{1}$ This syndrome is characterized by progressive muscle atrophy. ${ }^{2}$ Wasting and weakness start in the distal muscles of the lower limbs, and slowly spread proximally to appear in the hands and forearms over several years. ${ }^{1,2}$ Patients with CMTD exhibit an "inverted champagne bottle" appearance and motorsensory disorders in all 4 limbs. ${ }^{3}$ We report the case of a patient with CMTD who underwent dental implantation under moderate sedation in a dental clinic.

Received February 15, 2015; accepted for publication August 15, 2015

Address correspondence to Dr Naohiro Ohshita, Department of Anesthesiology, Osaka Dental University, 1-5-17, Ohtemae, Chuo, Osaka, 540-0008, Japan; oshita@cc.osaka-dent.ac.jp.

Anesth Prog 63:80-83 2016

(c) 2016 by the American Dental Society of Anesthesiology

\section{CASE REPORT}

A 51-year-old man $(170 \mathrm{~cm}, 71 \mathrm{~kg})$ with CMTD was scheduled for extraction and dental implantation under moderate sedation. We planned to perform the procedure in the dental clinic and to implant 9 fixtures into the maxillary and mandibular bones by using NobelGuide for immediate loading. The anesthetic method was selected by taking into account the degree of discomfort expected with the procedure and the desirability for analgesia and sedation for the patient, as well as the expected operation time of over 3 hours. At the time of the procedure, the patient's feet showed high arches and claw toes and fixation of both ankles (Figure, A). His hands showed wasting of the fingers with movement skill and sensory disorders (Figure, B). The patient had arthralgia, and the joints of his neck and shoulders tended to be stiff. During procedures, he often requested a break to stretch and exercise his neck, arms, and legs. 


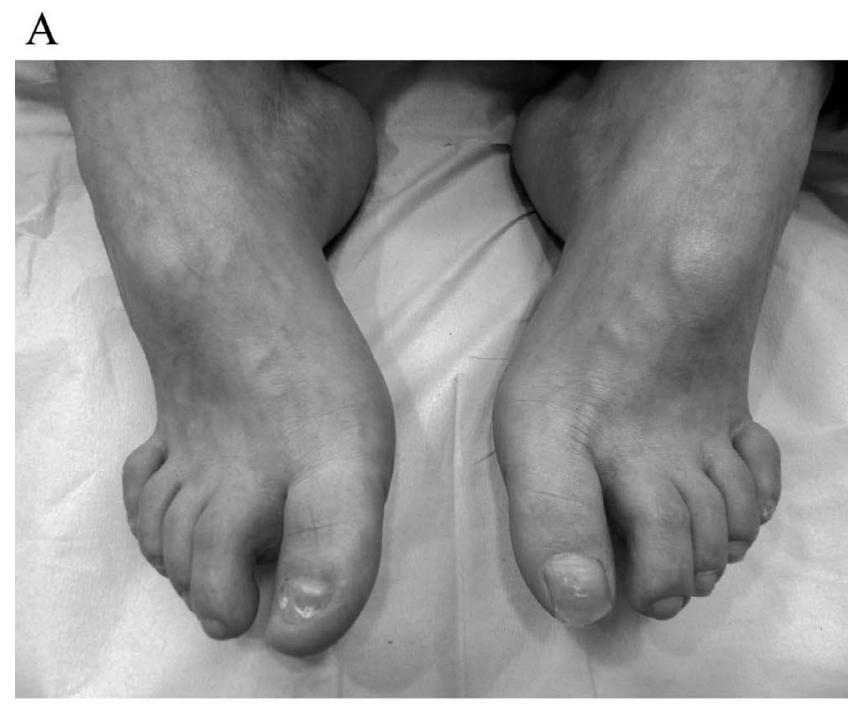

B

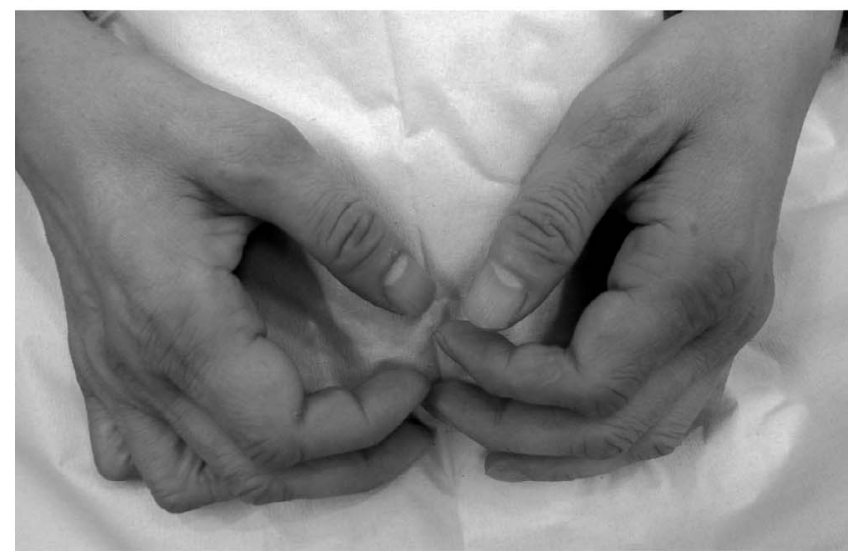

(A) The patient's feet showing high arches and claw toes. (B) Wasting of the fingers of the patient's hands.

His medical history also included hypertension, which was being treated with amlodipine. He was a smoker and had tachypnea but no dyspnea and spoke with a lisp without anarthria. The findings of his preoperative workup were normal, except for mild elevations in aspartate aminotransferase at $41 \mathrm{IU} / \mathrm{L}$ and alanine aminotransferase at $49 \mathrm{IU} / \mathrm{L}$ as well as an expected elevated creatine kinase at $515 \mathrm{IU} / \mathrm{L}$ on screening blood tests and forced expiratory volume percentage in 1 second of $50.3 \%$ on pulmonary function test.

On the day of the operation, the patient's vital signs were follows: blood pressure, 149/83 $\mathrm{mmHg}$; heart rate, 90 beats per minute with normal sinus rhythm; and oxygen saturation as measured by pulse oximetry, $96 \%$ on room air. He was given $1.5 \mathrm{mg}$ midazolam initially by a dental anesthesiologist, and assessments of sedation level according to the Ramsay sedation scale yielded a score of 3. Subsequently, propofol was infused continuously (10-30 $\mu \mathrm{g} / \mathrm{kg} / \mathrm{min}$; total dose, $300 \mathrm{mg})$. During the operation, the Ramsay score could be maintained at level 3 and vital signs were stable, with a systolic blood pressure of $120-140 \mathrm{mmHg}$, heart rate of $70-85$ beats per minute, and oxygen saturation as measured by pulse oximetry of $95-97 \%$ on room air during the invasive procedure. The surgeon administered $9 \mathrm{~mL}$ of the local anesthetic (2\% lidocaine including epinephrine $1: 80,000)$. We administered midazolam initially and propofol continuously, with total doses of $1.5 \mathrm{mg}$ and $300 \mathrm{mg}$, respectively, and completed the procedure in 3 hours and 30 minutes without adverse events.

\section{DISCUSSION}

CMTD was described simultaneously by J. M. Charcot, P. Marie, and H. H. Tooth in $1886 .{ }^{1}$ The incidence of CMTD is 1 in 2500-1 in 10,000, and there are more men affected than women. ${ }^{1}$ It is the most common inherited neurologic disorder in the United States. The chief features of CMTD are atrophy of the peroneal muscles and motor-sensory disorders in 4 limbs. ${ }^{1,2}$ However, the patterns of nerve degeneration and clinical symptoms vary, and CMTD is classified into 4 types: types 1 and 2 have autosomal-dominant inheritance, and the other types are recessive and have $\mathrm{X}$-linked inheritance. ${ }^{1}$ Life expectancy is normal.

CMTD is a peripheral neuropathy present in childhood that usually becomes clinically evident by the middle teenage years. Muscle wasting and weakness start in the lower limbs and slowly spread proximally to appear in the hands and forearms. ${ }^{2}$ Autonomic dysfunction is also commonly present. Myelin dysfunction and axonal degeneration are the main pathophysiologies, presenting with different clinical characteristics. This patient was diagnosed with poliomyelitis in childhood and an undefined peripheral nerve disorder in his later teenage years, even though his father was suspected of having CMTD. The quality of life of CMTD patients is affected by muscle strength (the degree of dysfunction in the lower limbs), aging, and the disease stage (the duration of the disease). ${ }^{4,5}$ Because quality of life can be dramatically influenced by physical limitations, patients with CMTD appear to be more susceptible to alterations in quality of life and present a higher risk of depression. ${ }^{4}$ This patient could ride a bicycle by himself and appeared healthy. However, his feet had the characteristic deformity and both ankles were fixed; in addition, his fingers showed wasting as well as skill movement and sensory disorders. He also had arthralgia, and the joints of his neck and shoulders tended to be stiff and often could not move significantly for several months at a time. During dental procedures, he often requested a break to 
stretch and exercise his neck, arms, and legs, the latter possibly associated with restless legs syndrome. ${ }^{6}$

Complications in general anesthetic treatment for CMTD patients are related to the neurologic disorder caused by myelin or axonal genes. Major considerations for the patients with CMTD are an increased sensitivity to nondepolarizing muscle relaxants and the risk of malignant hyperthermia. ${ }^{3,5,7-9}$ We chose to provide moderate sedation, because the NobelGuide implant procedure in this case would be a minimally invasive operation technique in which good local anesthesia could be obtained and thus moderate sedation was deemed sufficient.

We considered that potential problems with moderate sedation in the present CMTD patient included dysfunction of the autonomic nervous system, reduced perioperative respiratory function, sensitivity to intravenous anesthetic agents, and difficulty in positioning. Dysfunction of the autonomic nervous system might have resulted in hemodynamic change to the anesthetics, including sensitivity to intravenous agents and abnormalities of the electrocardiogram or dysrhythmia. Even if there are few conclusive publications documenting the disadvantage of the use of intravenous anesthetics in CMTD patients, many anesthesiologists raise serious concerns over this approach. However, recent reports of anesthetic managements for patients with CMTD have indicated that intravenous anesthesia would be safe and use of thiamylal, thiopental, and propofol would be successful. $^{2,10-12}$ Conscious sedation in neurosurgery requires propofol infusion rates of $<50 \mu \mathrm{g} / \mathrm{kg} / \mathrm{min}$. ${ }^{13}$ However, in this case, a rate of $10-30 \mu \mathrm{g} / \mathrm{kg} / \mathrm{min}$ was safe and effective for moderate sedation without hemodynamic change. Additionally, we administered midazolam cautiously and this patient exhibited a relaxed feeling (level 3 on the Ramsay sedation scale) with a small dose of midazolam $(1.5 \mathrm{mg})$.

Bispectral index monitoring would be effective for assessment of moderate sedation and for the patient's lack of motor responsiveness. However, the patient in this case often stretched and exercised his neck, arms, and legs, and these motions might be a disadvantage for bispectral index monitoring. Bispectral index value would elevate, at least in part because of electromyographic artifact, and moving the head commonly results in elevated electromyographic activity. ${ }^{14}$ We could manage this case using assessment of the Ramsay sedation score and tried to do moderate sedation. In addition, we prepared for basic and advanced life support for potential emergencies with the family physician's cooperation.

Anesthesia-related cardiovascular and respiratory dysfunction would be important issues to consider in this case. Concerning abnormalities of the electrocardio- gram, there were no problems in this case with sedation or after injection of the local anesthetic (2\% lidocaine including $1: 80,000$ epinephrine). Previous reports suggest that patients with CMTD may be affected by several types of dysrhythmia in the medical history, including complete right bundle branch block, mitral valve prolapse with second-degree atrioventricular block, third-degree atrioventricular block, sick sinus syndrome requiring pacemaker insertion, and paroxysmal atrial flutter. ${ }^{3,15}$ Other reports of arrhythmic events during surgical procedures suggest that premature ventricular contraction gives rise to monofocal or multiple QT prolongation. ${ }^{5,15}$

Concerning respiratory function, patients with CMTD may have disorders of diaphragmatic function, abnormalities of the thorax cage leading to restricted pulmonary function, and sleep disorders. ${ }^{4,8,9}$ Respiratory muscle weakness may have debilitating and serious consequences, and patients have been reported to develop pneumonia or require extended ventilation for up to 1 month postoperatively. ${ }^{7}$ In addition, patients with CMTD may have difficulties lying in the supine position because of an abnormal thoracic cage or orthopnea. ${ }^{8,9}$ Pulmonary function testing, including forced vital capacity (FVC), percentage of predicted value (\%FVC), and postural changes in \%FVC, are clinically useful measures for evaluating patients with CMTD. In the present case, the \%FVC value of 125 was within normal limits, but the forced expiratory volume percentage in 1 second value of 50 was significantly low, and the patient exhibited symptoms of obstructive pulmonary function. We considered that the forced expiratory volume percentage in 1 second value was related to smoking, although the cause of tachypnea was unknown. Respiratory depression may be related to disorders of the cranial nerves IX, X, and XI, and these disorders may also reduce the swallowing reflex and paralyze the vocal cords, leading to postoperative respiratory complications. ${ }^{6}$ In the present case, disorders in the central nervous system might have caused the tachypnea and lisp, both possible symptoms in patients with CMTD.

In conclusion, we found that moderate sedation using intravenous propofol and midazolam for oral surgery was a safe and effective anesthetic method for this patient with CMTD.

\section{REFERENCES}

1. Timmerman V, Strickland AV, Züchner S. Genetics of Charcot-Marie-Tooth (CMT) disease within the frame of the Human Genome Project success. Genes. 2014;22:13-32. 
2. Harding AE, Thomas PK. The clinical features of hereditary motor and sensory neuropathy types I and II. Brain. 1980;103:259-280.

3. Roelofse JA, Shipton EA. Anaesthesia for abdominal hysterectomy in Charcot-Marie-Tooth disease. A case report. $S$ Afr Med J. 1985;67:605-606.

4. Cordeiro JL, Marques W, Hallak JE, Osório FL. Charcot-Marie-Tooth disease, psychiatric indicators and quality of life: a systematic review. ASN Neuro. 2014;27:185-192.

5. Schmitt HJ, Münster T. Mivacurium-induced neuromuscular block in adult patients suffering from Charcot-MarieTooth disease. Can J Anaesth. 2006;53:984-988.

6. Aboussouan LS, Lewis RA, Shy ME. Disorders of pulmonary function, sleep, and the upper airway in CharcotMarie-Tooth disease. Lung. 2007;185:1-7.

7. Antognini JF. Anaesthesia for Charcot-Marie-Tooth disease: a review of 86 cases. Can J Anaesth. 1992;39: 398-400.

8. Pasha TM, Knowles A. Anaesthetic management of a patient with Charcot-Marie-Tooth disease for staged diaphragmatic plication. Br J Anaesth. 2013;110:1061-1063.
9. Nathanson BN, Yu DG, Chan CK. Respiratory muscle weakness in Charcot-Marie-Tooth disease. Arch Intern Med. 1989; 149:1389-1391.

10. Greenberg RS, Parker SD. Anesthetic management for the child with Charcot-Marie-Tooth disease. Anesth Analg. 1992;74:305-307.

11. Naguib M, Samarkandi AH. Response to atracurium and mivacurium in a patient with Charcot-Marie-Tooth disease. Can J Anaesth. 1998;45:56-59.

12. Schmitt HJ, Huberth S, Huber H, Munster T. Catherterbased distal sciatic nerve block in patients with Charcot-MarieTooth disease. BMC Anesth. 2014;14:8.

13. Venkatraghavan L, Luciano M, Manninen P. Anesthetic management of patients undergoing deep brain stimulator insertion. Anesth Analg. 2010;110:1138-1145.

14. Fraser GL, Riker RR. Bispectral index monitoring in the intensive care unit provides more signal than noise. Pharmacotherapy. 2005;25:19S-27S.

15. Tetzlaff JE, Schwendt I. Arrhythmia and Charcot-MarieTooth disease during anesthesia. Can J Anaesth. 2000;47: 829. 